\title{
Le manuel Corso di Lingua francese a base intuitiva de Romeo Lovera
}

The manual Corso di Lingua francese a base intuitiva by Romeo Lovera

\section{Marie-Denise Sclafani}

\section{(2) OpenEdition}

Édition électronique

URL : https://journals.openedition.org/dhfles/7829

DOI : $10.4000 /$ dhfles.7829

ISSN : 2221-4038

Éditeur

Société Internationale pour l'Histoire du Français Langue Étrangère ou Seconde

Édition imprimée

Date de publication : 1 décembre 2020

Pagination : 161-181

ISSN : 0992-7654

Référence électronique

Marie-Denise Sclafani, « Le manuel Corso di Lingua francese a base intuitiva de Romeo Lovera »,

Documents pour l'histoire du français langue étrangère ou seconde [En ligne], 64-65 | 2020, mis en ligne le 01 décembre 2020, consulté le 29 mars 2023. URL : http://journals.openedition.org/dhfles/7829;

DOI : https://doi.org/10.4000/dhfles.7829

Ce document a été généré automatiquement le 29 mars 2023.

Tous droits réservés 


\section{Le manuel Corso di Lingua francese a base intuitiva de Romeo Lovera}

The manual Corso di Lingua francese a base intuitiva by Romeo Lovera

Marie-Denise Sclafani

\section{Introduction}

1 Nous nous proposons d'analyser les trois volumes du manuel de Romeo Lovera, Corso di Lingua francese a base intuitiva (1902-1936), destinés à l'enseignement de la langue française dans les trois premières années de l'école secondaire en Italie.

2 Tout d'abord, nous prendrons en considération la volonté d'un groupe d'enseignants de langues étrangères en Italie, manifestée dans les pages de la revue Bollettino di Filologia Moderna (dorénavant BFM), d'adopter les nouvelles méthodologies et pratiques didactiques pour l'enseignement des langues vivantes. L'attention se focalisera ensuite, d'une part, sur Romeo Lovera (1861-1922), fondateur du BFM, qui sera l'âme de ce changement, et d'autre part sur son manuel, qui à l'orée du lent glissement vers des pratiques plus modernes d'enseignement des langues vivantes en Italie, appliquera les nouvelles méthodes, en particulier, la méthode intuitive, en offrant de cette manière un souffle actuel et frais là où la tradition, liée à l'enseignement des langues vivantes sur le modèle grammaire-traduction, persistait encore.

\section{Cadre historique et enseignement des langues étrangères}

3 Le débat en Europe sur l'enseignement des langues modernes s'étend et s'enflamme durant une période historique de forts changements politiques et économiques; il s'agit de la fin du XIX ${ }^{e}$ siècle, une période où le développement de l'industrie et l'intensification des relations commerciales contribuent à la prise de conscience de la nécessité pressante d'un enseignement/apprentissage plus complet et pratique des 
langues étrangères. Apprendre une langue vivante, de manière pratique, devient, donc, un élément indispensable de succès dans le monde contemporain. Cette idée commence à s'enraciner à tous les niveaux de l'éducation scolaire.

C'est une réaction à la méthode dite "classique ", centrée sur la prédominance de l'enseignement écrit au détriment de la pratique orale, sur l'apprentissage de la langue par la grammaire, de manière déductive et sur la traduction de phrases sans aucun lien entre elles, qui sont fortement artificielles et peu attentives à l'authenticité de la langue.

5 L'attention des opposants aux vieilles méthodes, considérées comme dépassées et inefficaces, se focalise sur une méthode dite naturelle, maternelle, intuitive, directe qui offre un changement de direction à l'enseignement des langues vivantes en Europe. Le point de départ à cette innovation «nous vient de l'Allemagne où, en 1882, paraît le pamphlet d'un professeur de philologie anglo-saxonne de l'Université de Marbourg, Wilhelm Viëtor, qui porte un titre éloquent: Der Sprachunterricht muss umkehren (l'enseignement des langues doit changer radicalement) $»^{1}$, (Pellandra 2004 : 90 ; Galazzi 1990: 296). Le double souci des novateurs est de rendre l'enseignement plus intéressant, plus attrayant pour l'élève (Deniker $1904: 49$ ).

\section{Romeo Lovera : L'homme et ses publications}

6 En Italie, c'est Lovera qui divulgue, par le biais de la revue le $B F M$, qu'il fonde et dirige, les nouvelles théories, les méthodes scientifiques et leurs applications didactiques. Lovera, fin «observateur de la situation de l'enseignement des langues vivantes hors d'Italie, interlocuteur des réformistes les plus actifs» (Minerva $2005: 8$ ), est, donc, le plus grand partisan de la réforme méthodologique de l'enseignement des langues étrangères. Dans ses articles, il prône la méthode intuitive, tout en critiquant les programmes ministériels obsolètes et incomplets adoptés par l'école italienne et le vieux système déductif ou grammatical d'enseignement des langues.

7 Lovera, enseignant polyglotte, publie de nombreux ouvrages, manuels et livres de grammaire en allemand, français, roumain et grec moderne pour l'enseignement/ apprentissage des langues vivantes; il traduit aussi, de l'allemand, des œuvres de littérature, d'histoire, de philosophie et d'économie (Lentini 2012: 484). Sa grande expérience dans l'enseignement des langues lui permet d'être choisi pour diriger, en 1904, la toute nouvelle Scuola di Commercio à Palerme. C'est une charge qu'il estime «utile et qualifiante pour porter à terme son projet didactique $»^{2}$ (Lentini $\left.2012: 502\right)$ et qu'il accepte dans l'espoir de pouvoir atteindre un autre objectif : devenir professeur d'université à Palerme, un poste qu'il n'obtiendra pas. Il est, en outre, membre de l'Association Phonétique Internationale, dont il devient Conseiller de 1904 à 1910, et premier président de l'Association italienne des professeurs de langues étrangères (Galazzi 2002: 16).

8 La revue, le $B F M^{3}$, connaît deux phases : la première de 1894 à 1896, publiée à Salò, et la seconde de 1901-1909, publiée jusqu'en 1904, d'abord, à Venise et ensuite à Palerme (Rossignotti 1991 : 265-266). Lovera fonde, en 1910, L'écho français, qui devait en être la continuation et paraitre deux fois par mois ; mais la revue durera peu.

9 Le but de la revue fondée par Lovera, le BFM, est explicité dans les pages du premier numéro, paru le 15 janvier 1894. En premier lieu, le Bollettino, rédigé par des 
enseignants, "a l'intention de présenter au public [...] une liste des publications philologiques [...] en ajoutant leurs propres appréciations inspirées toujours de la plus sincère conviction et de l'utilité pratique que l'on devrait tirer des langues étrangères ${ }^{4}$, ensuite, de rassembler le plus grand nombre d'enseignants afin de résoudre certaines questions non résolues dans l'école italienne, telles que la discussion sur les programmes scolaires, sur les manuels scolaires et sur la position des enseignants à l'école, sous-payés et souvent dans une situation précaire (Rossignotti 1991 : 270). Il s'agit d'un appel plein d'espoir pour que tous ceux, qui demandent un enseignement des langues plus rationnel et qui ont à cœur « la langue vivante et non fossilisée $»^{5}$, puissent s'unir et réclamer aux autorités les réformes nécessaires.

Le Bollettino joue un rôle décisif et diffuse les idées nouvelles en Italie, là, où les défenseurs des vieilles méthodes s'accrochaient à la tradition sans aucune intention de remettre en question la validité de la méthode d'enseignement des langues vivantes: « la méthode directe, à cause de son empirisme, n'est utile, selon l'avis de plusieurs, que pour les débutants ou pour un enseignement pratique » (Minerva 1996 : 155).

11 En 1905, le ministre Bianchi, dans le but de renouveler les programmes obsolètes d'enseignement des langues étrangères de l'école italienne, préconise l'introduction de la méthode directe, naturelle et intuitive pour l'apprentissage de la langue orale, l'étude des sons selon les principes de la phonétique et l'enseignement grammatical inductif. C'est une première prise de position en faveur des réformateurs, mais, en 1908, l'on est bien loin, encore, de cet objectif. Lovera explique les raisons qui empêchent l'introduction totale de la méthode directe dans les écoles italiennes: « La méthode directe requiert une attention constante, de la part des élèves, et une connaissance profonde de la langue étrangère de la part des enseignants : deux bonnes qualités qui font encore défaut en Italie $»^{6}$.

\section{Romeo Lovera et ses théories sur l'enseignement du français}

12 "Avant tout il faut apprendre à parler, puis à lire, enfin à écrire "7. Il s'agit, pour Lovera, du concept de l'enseignement des langues étrangères. Dans un article Il metodo grammaticale-traduttivo ed il metodo intuitivo (Lovera 1901b : 2) il défend les réformateurs, en s'insérant parmi eux, et insiste sur le fait qu'ils ne proposent rien de bien nouveau, car les bases de la méthode directe remontent à plus de deux siècles; on en trouve des traces dans les œuvres de pédagogues et de philosophes tels que Bacon, Comenius, Rousseau d'une part, et Tommaseo, Pestalozzi, Ferrante Aporti d'autre part. Selon Lovera, parmi les nouvelles méthodes d'enseignement linguistique, celles qui ont une possibilité majeure d'être accueillies et acceptées dans les écoles et par les enseignants, sont la méthode Börner, la méthode Gouin et la méthode intuitive. Toutes les trois peuvent être adoptées, soit singulièrement soit fusionnées entre elles; elles ont toutes l'objectif de conduire les élèves à parler et, en deuxième lieu, à écrire la langue étrangère avec un minimum d'effort pour un apprentissage moins mnémonique et plus éducatif et instructif. Ces méthodes, quoiqu'apparemment en opposition entre elles, possèdent toutes une base commune "dans les principes qui guident l'association phonétique, dont le président Vietör [...] est le plus ardent défenseur de la réforme de l'enseignement linguistique $»^{8}$. 
13 Lovera précise, dans la préface du premier volume de son manuel ${ }^{9}$, que l'« enseignement doit être essentiellement oral [...]. Avant de frapper l'œil de l'élève avec l'écrit, il faut frapper son oreille avec le son ». Ainsi, dès la première leçon, à travers l'intuition directe et indirecte de la langue étrangère, l'élève pourra commencer à parler la langue française en étudiant les expressions et les phrases les plus usuelles et en apprenant la grammaire de manière inductive. Il s'agit donc de l'application de la méthode orale directe tournée vers la pratique de la langue, la méthode intuitive ou directe. Dans ses manuels, la langue maternelle (LM) est très peu présente.

\section{Romeo Lovera et l'application de la méthode qu'il prônait dans le Corso di lingua francese a base intuitiva}

14 «Au premier coup d'œil, on est frappé de l'art et du soin de l'exécution matérielle de l'ouvrage [...]. Dès la première ligne, on se sent en présence d'un homme pratique, qui connaît l'enseignement, qui possède 'sa classe dans sa main' ». C'est avec ces termes élogieux que Léonard (ou Léon) Chambonnaud présente, dans les pages du premier numéro de L'écho français, publié le 15 janvier 1910 à Palerme, le manuel de Romeo Lovera arrivé à sa quatrième édition. Le manuel est publié pour la première fois en 1902 à Venise par la Libreria editrice del Bollettino di Filologia Moderna, avec la mention « ediz. A. per le scuole maschili». Nombreuses seront les rééditions de son manuel (la dernière édition attestée date de 1936) et vaste en est aussi sa diffusion dans les écoles publiques $^{10}$ de la jeune République italienne ${ }^{11}$.

\section{Prononciation et orthographe}

Le manuel comporte trois volumes qui correspondent à trois années d'enseignement du français. Seule la première partie du premier volume est consacrée à la prononciation et à l'orthographe; ce choix reflète le fait que dans les manuels qui appliquent la méthode directe «La prononciation est prise en compte dès les débuts de l'apprentissage de L2 " (Germain 1993 : 129). Il est important de mettre en évidence, aussi, qu'un des articles fondamentaux (notamment le troisième) du programme de l'Association Phonétique Internationale, explicité, entre autres, dans un article du BFM de 1901, précise : «Le premier soin de l'enseignant doit être celui de familiariser les élèves aux sons de la langue étrangère. Pour atteindre cet objectif, il recourra à une transcription phonétique, qui sera utilisée à la place de l'orthographe traditionnelle pendant la première partie du cours $»^{12}$ (Lovera 1901: 7). C'est donc ce que Lovera choisit d'appliquer dans le premier volume de son Corso. Cette première partie a été spécialement conçue à l'usage des enseignants qui pourront utiliser les règles, rassemblées dans un tableau, en classe, s'ils choisissent d'enseigner l'orthoépie en parallèle avec la langue orale.

\section{Exercices d'intuition pour la pratique de l'oral}

Dans ses articles, il met l'accent, en particulier, sur la méthode intuitive, qui est un processus qui agit sur les sens, notamment sur la vue (Lovera 1901a :22). Cette méthode, dont il spécifie qu'il ne s'agit pas d'une nouveauté, car elle a déjà été utilisée comme soutien pour l'enseignement de la LM, permet de parler du monde qui entoure l'élève 
sans avoir recours à la LM. "L'enseignement intuitif [...] est un avantage incalculable pour le progrès des échanges intellectuels et commerciaux des nations civilisées $»^{13}$ (Lovera 1909 : 16).

En cohérence avec l'application de l'intuition directe, qui s'exerce sur la réalité ou sa représentation figurée, Lovera insère, dans la première partie des trois volumes de son manuel, une série d'exercices d'intuition (leçons de choses), conçus pour que l'enseignant puisse présenter à la classe des sujets de la vie courante de manière directe sans avoir recours à la LM et par le biais desquels les élèves « saisissent la signification des mots à travers la réalité qui les entoure et les exemples » (Finotti $2010: 3$ ). Pour ce faire, il aura besoin d'avoir à sa disposition tous les objets présentés dans les différents exercices ou, sinon, dans l'impossibilité de pouvoir montrer physiquement «un vaste champ d'observation ", il aura recours aux tableaux muraux ${ }^{14}$, qui représentent les différents moments de la vie humaine courante : «Les tableaux muraux pour l'enseignement des langues sont peu répandus chez nous; ils commencent à être introduits dans les écoles, et l'Italie peut compter sur les professeurs Lovera et Manfredini, qui sont en train d'en publier une série afin d'atteindre cet objectif $»^{15}$ (Ravà 1907 : 61). Il s'agit des tableaux muraux de Hölzel ${ }^{16}$, déjà utilisés dans les écoles d'autres pays en Europe, à savoir, la Croatie, la Belgique, le Danemark, la Suisse et la Suède (Rossignotti 1990 : 271), pour l'application de l'intuition indirecte qui suggère la réalité grâce à des procédés tels que : le geste, la mimique, l'intonation, les mouvements, etc. (Puren 1988: 96). La reproduction, en petit, des tableaux muraux est insérée même à la fin de chaque volume afin que l'élève "puisse s'en servir pour des répétitions à domicile » (Lovera 1903a: 7 "enseignement intuitif»). Les volumes présentent, donc, de nombreux chapitres, subdivisés à leur tour en sous-chapitres : La vie scolaire (Premier cycle : Les objets de la salle d'école, Les personnes dans la salle d'école, La classe de dessin et de couture, Qualités et usages des objets de la classe, Les leçons, Notre maison d'école; Deuxième cycle: L'homme; L'homme et les choses, Le corps humain, Les vêtements des écoliers, La famille, Le temps) et L'habitation à la ville (La maison d'habitation, La salle à manger, Le salon, La cuisine). Le deuxième volume: L'habitation à la campagne (La ferme, La laiterie, L'écurie, Le village), La ville (La place, La gare, Les grands magasins, La foire, L'instruction publique) et L'Europe. Le troisième volume : La nature (Le port de mer; Une nuit d'été en mer, Collines et lacs, Le (sic) haute montagne). Quelques extraits :

Premier volume :

I. Les objets de la salle d'école

II. Exercice. - Voici un livre, le livre. Qu'est ceci ? C'est un livre, le livre. Voici une plume, la plume. Qu'est ceci? C'est une plume, la plume. La plume est un objet d'école. [...].

III. Les personnes dans la salle d'école.

III. Exercice. - Je suis le professeur et tu es un élève. Tu es un élève, il est un élève. Nous sommes le professeur et les élèves. Vous êtes des élèves. Ils sont des élèves. Où sommes-nous? [...]. (1907: 17et 19, Exercices $n^{\circ}$ I et $n^{\circ}$ III).

La salle à manger

\section{Coup d'œil d'ensemble}

XXVIII. Exercice. - Cette scène nous transporte dans la salle à manger d'une bonne famille bourgeoise. Comme toute chambre elle a quatre murs, mais nous n'en voyons que trois. Les murs sont tendus de papier vert. A gauche on aperçoit une grande fenêtre tendue de rideaux. A droite il y a un poêle-cheminée en faïence, surmonté d'une glace avec un cadre en bois. [...]. (1907 : 72). 


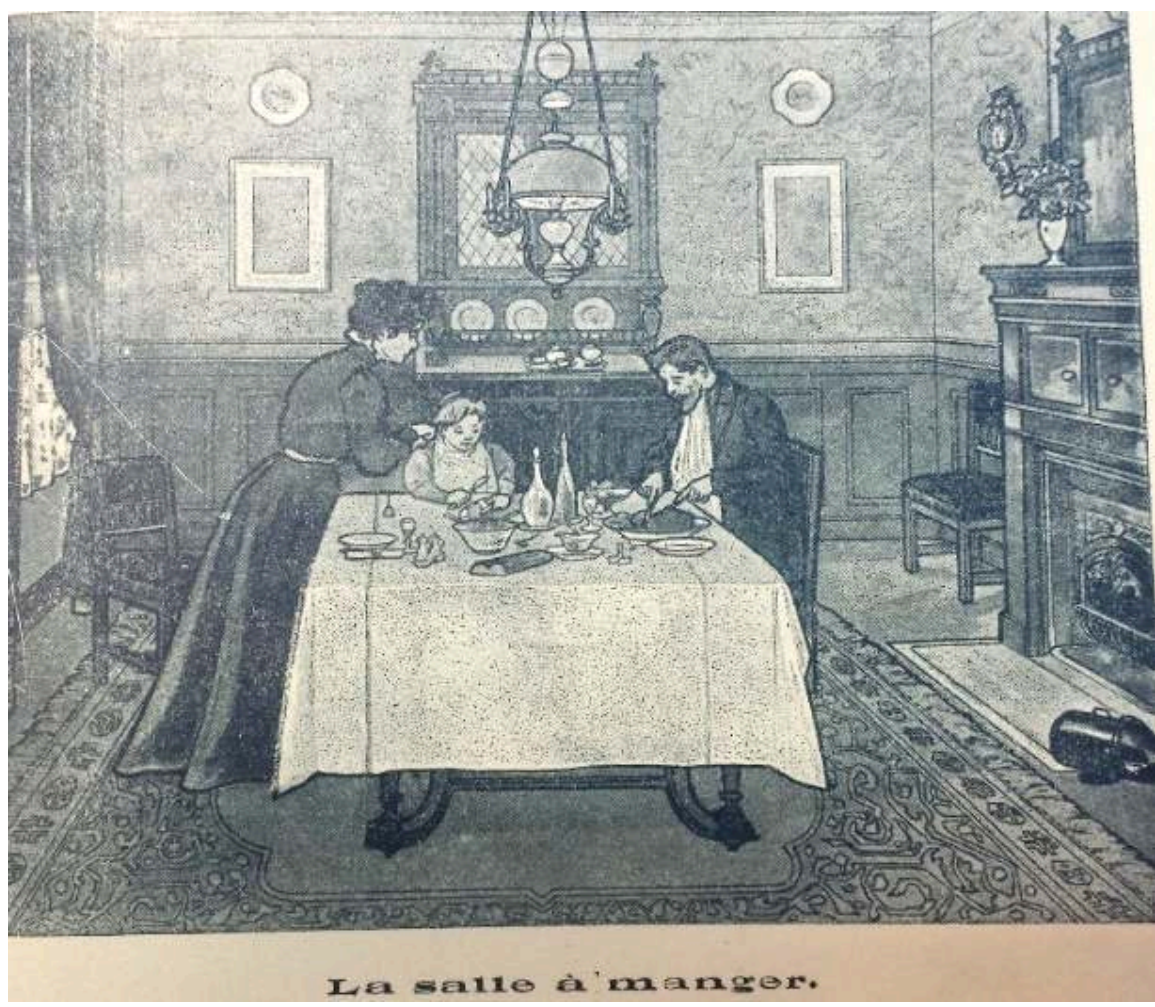

Document 1 : La salle à manger (1907)

Lovera recommande, dans la préface, que l'exercice soit fait oralement et que les élèves aient les livres fermés pendant l'exposition. Pour qu'ils puissent acquérir une bonne prononciation, ils doivent avant tout écouter. L'enseignant doit donc exposer l'exercice phrase après phrase, en s'aidant par le geste ou avec les objets qu'il a, à sa disposition. Tous les termes nouveaux sont graphiquement mis en évidence en caractères gras pour attirer l'attention des élèves. Les nouveaux termes sont introduits petit à petit de manière graduelle. En ce qui concerne les deux premiers exercices (I et III), l'enseignant lit les phrases en présentant physiquement les objets présents dans la salle; pour le troisième exercice (XXVIII) il présente, au contraire, l'image. Qu'il s'agisse de l'enseignement intuitif direct ou de l'enseignement intuitif indirect, les termes sont appris par le biais de petites phrases qui ont un sens accompli et qui sont reliées, les unes aux autres, par un sens commun, évitant de cette manière la mémorisation de longues listes de termes rares et non usités dans la vie de tous les jours.

Deuxième volume :

\section{L'ÉCURIE}

\section{I- L'intérieur de l'écurie}

VII. Exercice. - Cette scène nous transporte dans une écurie où logent chevaux, mulets et ânes. Plusieurs garçons de ferme sont occupés à panser et à harnacher des chevaux. [...] A droite on voit un charretier en blouse, le fouet passé sur l'épaule, qui enlève le harnais d'un gros cheval blanc. Il vient de faire un tour dans les champs. [...]. (1919: 16, Exercice $\mathrm{n}^{\circ}$ VII). 


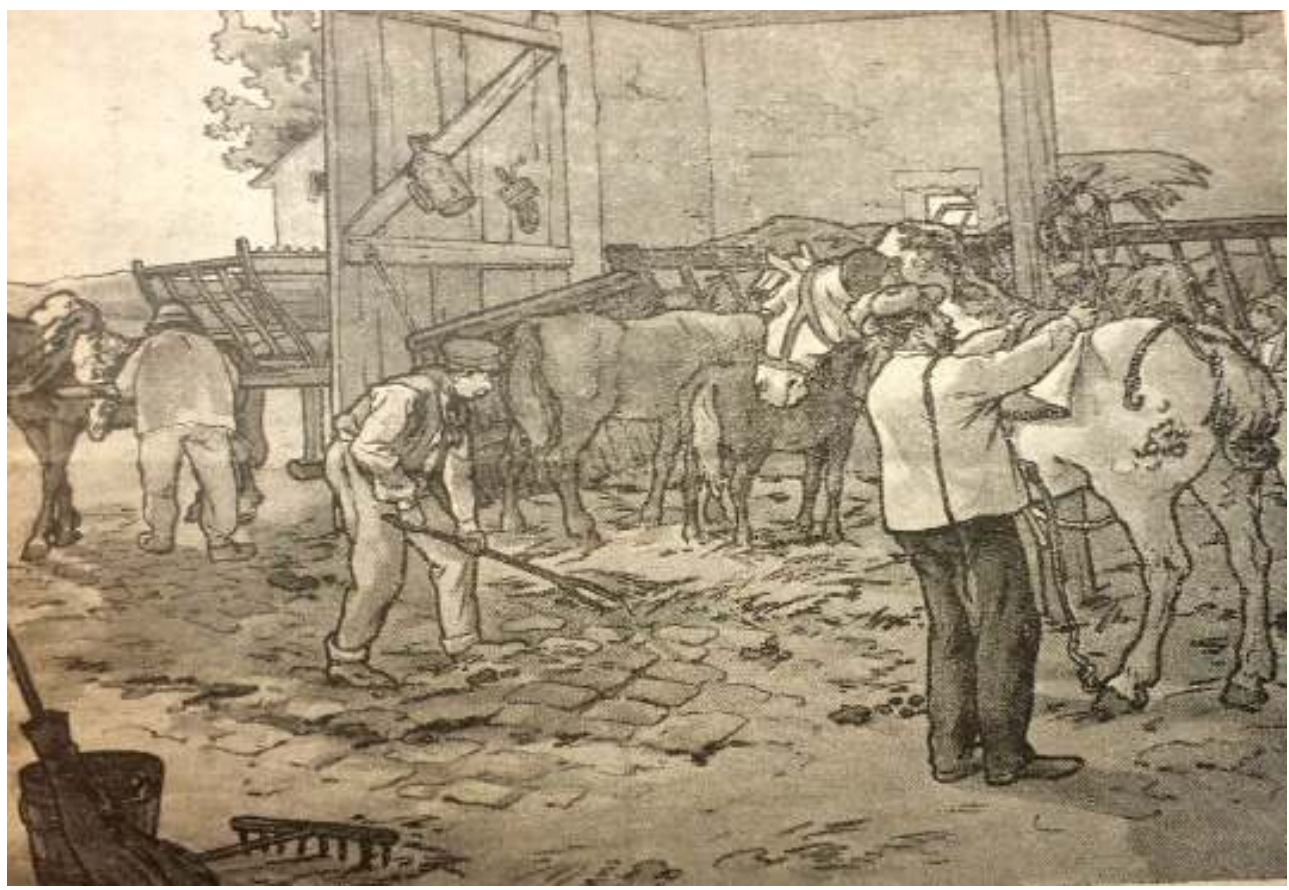

Document 2 : L'« écurie »

Troisième volume :

LA NATURE

UNE NUIT D'ÉTÉ EN MER

I- La mer et la pêche

Exercice. - A. La deuxième image du tableau nous montre la mer [...] dans le calme d'une belle nuit d'été. Le ciel est tout émaillé d'étoiles et la lune qui brille au milieu de quelques légers nuages, éclaire toute la scène de ses pâles rayons. [...] Plusieurs bateaux sillonnent la mer; ce sont des bateaux qui reviennent (1) de la pêche. [...]. (1917 :12 Exercice n. I).

(1) On revient au lieu d'où l'on était parti. On retourne au lieu où l'on était allé. On revient dans sa patrie. On retourne dans son exil. 


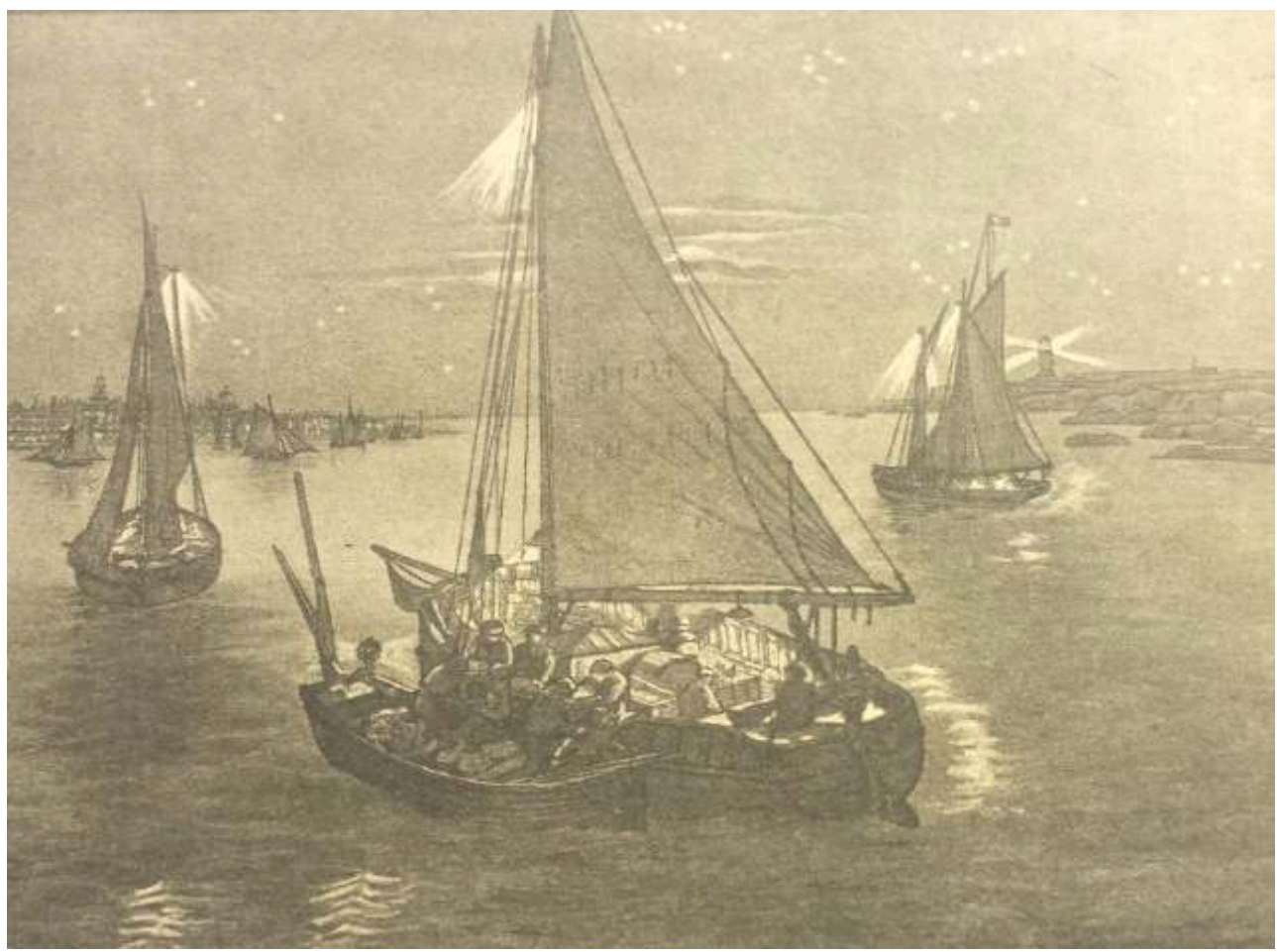

Document 3 : « une nuit d'été en mer »

Les exercices, ainsi composés, permettent à l'élève d'activer et d'affiner ses facultés de perception externe par le biais de l'association de la forme et du sens. Ils permettent en outre, de remplacer l'étude des mots par l'observation des choses, la mémoire par le jugement, la passivité par la participation active «[...] l'apprenant doit participer de façon active à son propre apprentissage : c'est ainsi qu'il doit répondre aux questions de l'enseignant, lire à voix haute les passages assignés, poser des questions, etc. » (Germain 1993 : 128). Les élèves acquièrent la capacité d'opérer des associations directes entre la langue étrangère et la réalité, sans devoir utiliser la LM. Tous les exercices sont suivis d'un Questionnaire qui sert de support aux enseignants et aux élèves en tant qu'exercice de conversation; un exercice qui se révèle très utile si l'enseignant maitrise la langue cible; c'est d'ailleurs ce que préconise Lovera dans la préface et dans tous les articles parus sur le $B F M$ quand il se réfère à la formation et à la préparation des enseignants. Le questionnaire pourra servir ensuite pour le travail de l'écrit. Voici un exemple de questionnaire qui suit le premier exercice « les objets de la salle d'école » :

Questionnaire. - Qu'est-ce que le tableau noir? Qu' est-ce que le crayon? Le livre est-il un objet d'école? Qu' est-ce que la plume? La craie est-elle un objet d'école?

Document 4 : Premier volume $(1907: 17)$

\section{La grammaire}

La grammaire n'est pas toutefois absente; elle suit le questionnaire et son apprentissage se fait de manière inductive (« des exemples aux règles » Puren 2012 [1988] : 28 et 96). L'on peut déduire donc les règles de grammaire des exercices: la 
méthode intuitive ne peut suffire, sans grammaire, à donner une connaissance complète de la langue "Sans elle [...] les phrases apprises sembleront à la mémoire les notes d'un air fugitif, que l'on reconnait quand on l'entend, dont on peut fredonner quelques mesures, mais que l'on se sent incapable d'exécuter " (Deniker 1904 : 52). C'est aussi la conception de Lovera: la grammaire est invisible mais, en même temps, toujours présente et, avec le Devoir incorporé à la fin de l'exercice d'intuition, elle peut être apprise ou reprise les derniers mois de l'année scolaire.

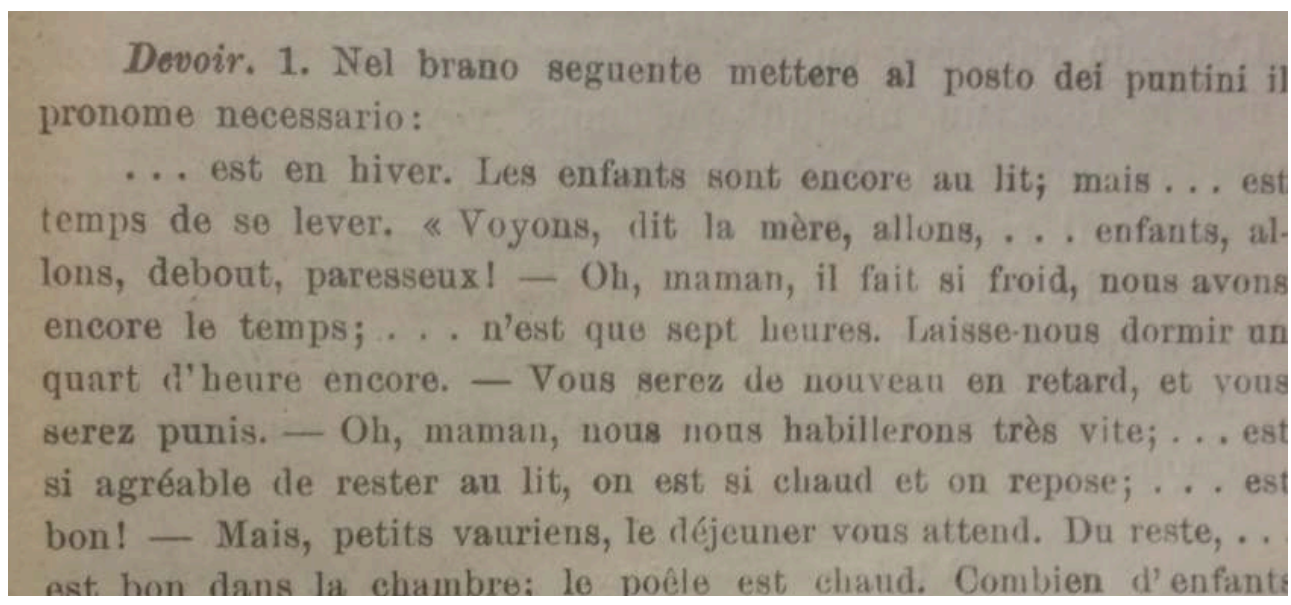

Document 5 : Deuxième volume (1919 : 25)

Dans cet exercice à trous, il faut insérer le pronom personnel sujet adéquat.

Des tableaux de synthèse de grammaire sont proposés pour les trois volumes; dans le premier, Lovera propose des tableaux qui résument la grammaire de la première année (la déclinaison de l'article défini et indéfini, l'article partitif, le pluriel des noms, le féminin des adjectifs, etc.), dans le deuxième l'on retrouve aussi «Les conjugaisons mortes ou archaïques », dans le troisième « La syntaxe des mots » et « La syntaxe de la proposition ». La grammaire doit être apprise de manière inductive « En acquérant, par induction, la grammaire des textes étudiés, on la rendra réellement utile et appréciée, car l'étudiant se rendra compte qu'il s'agit bien de la codification des lois du langage, et non pas d'une récolte de dogmes obscurs, éducateurs d'un peuple de perroquets $»^{17}$ (Lovera 1901: 10-11). La grammaire n'a plus un rôle central, elle n'est plus le point de départ, mais le résultat de l'enseignement grammatical. Un exemple, toujours du premier exercice :

Grammaire. - 1. In francese, come in italiano, vi soro due generi, il maschile e il femminile, e tre articoli, il determinativo, l'indeterminativo e il partitivo. - 2. L'articolo determinativo è: le davanti a un nome maschile singolare che comincia per consonante (o $h$ aspirata, v. Parte prima, $\S 14$ ): le livre, le crayon; la davanti a un nome femminile singolare che comincia per consonante $\left(o h_{b}\right.$ aspirata): la plume, la craie; l' davanti a un nome maschile o fem-

Lovera, Corso di lingua francese. Anno I. 3. Ediz.

Document 6 : Premier volume $(1907: 17)$ 

l'introduction de la lecture directe qui s'ajoute à l'intuition indirecte par le biais des tableaux muraux. C'est dans un article, "La Lettura diretta", publié sur le BFM que Lovera (1903b:140-143) explique que la lecture directe exige que l'enseignement de la langue soit fait sur la base de la méthode intuitive, qu'il considère comme la «base de l'édifice ", et que par le biais de la méthode directe après la lecture du texte, l'élève doit procéder à son interprétation en analysant tous les termes de manière spécifique et une phrase à la fois.

" La lecture directe » est, selon Lovera et Manfredini ${ }^{19}$, l'accomplissement et le pivot de tout enseignement linguistique. L'enseignant doit guider les apprenants, durant la lecture, en recourant le moins possible à la LM, et donc expliquer les termes inconnus au moyen de termes familiers et simples, en employant, si nécessaire, des synonymes et des circonlocutions. Le but de la lecture est de faire connaître aux élèves, l'histoire, la culture, la géographie, le caractère, l'évolution de la société et les habitudes du peuple qui utilise la langue objet de l'apprentissage : «[...] les contenus civilisationnels tenus pour indispensables à l'apprentissage linguistique entrent dans les manuels » (Minerva 2005 : 3). Voici un exemple : 


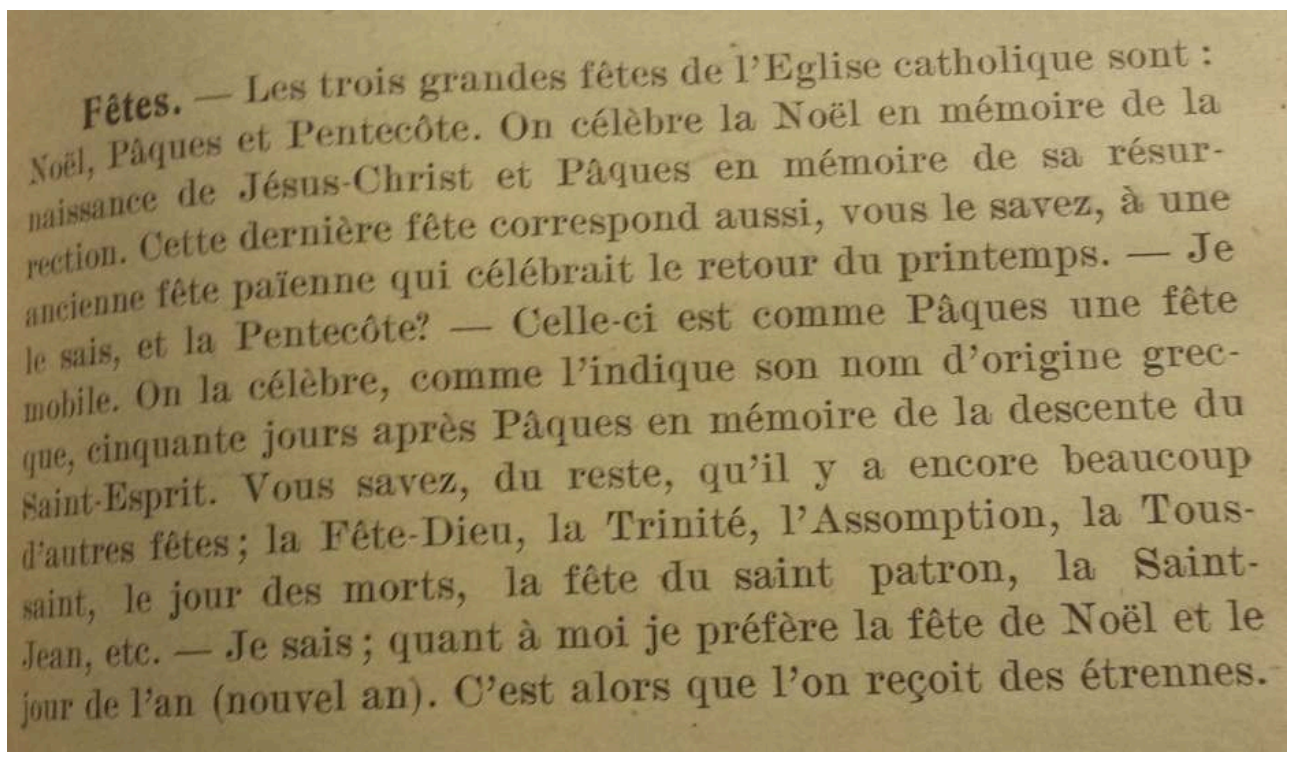

Document 7 : Troisième volume « Choix de lectures » $(1917: 95)$

31 L'exercice de lecture des textes, qui, au tout début de l'apprentissage, doivent nécessairement être simples et brefs, se déroule ainsi : l'enseignant lit en premier le texte et ensuite les élèves le relisent; durant la lecture, l'enseignant soulignera et corrigera les erreurs de prononciation. Les élèves, qui sont habitués à la méthode intuitive, dès la première lecture, comprennent le sens général du texte ; la plupart des termes leur sont familiers car ils ont été utilisés dans les lectures précédentes ou dans les autres exercices du manuel. Il s'agit de la même compétence que celle d'« un de nos enfants qui, de 8 ans à 10 ans, comprend son livre de lecture sans toutefois saisir tout le sens des détails $»^{20}$. La deuxième phase de la lecture consiste dans l'interprétation du texte : les termes abstraits seront donc expliqués en ayant recours aux idées concrètes (quelques exemples : factice $=$ artificiel, qui n'est pas réel ; se dépouiller $=$ perdre ce qui couvre; nuance = légère gradation de couleur, différence délicate entre choses du même genre) ou aux familles de vocables (père - paternel - paternité - patrie patriotique, etc.). Les autres termes, dont le sens reste opaque et qu'il est difficile d'expliquer par le biais de synonymes, seront traduits en LM, mais seulement quand "l'on est obligé de jeter sa langue aux chiens " "21. L'enseignant aura recours à une série de questions pour que l'élève puisse comprendre le sens profond de la lecture et ainsi diriger la conversation. Après ce travail d'interprétation, le texte ne présentera plus aucune difficulté pour l'élève, qui pourra le relire de manière fluide et non pas mécaniquement.

A la fin du dernier volume, Lovera, introduit aussi des exercices sur les synonymes.

\section{Thèmes}

Tout en critiquant âprement la méthode grammaire-traduction, Lovera se voit obligé d'insérer dans son manuel des thèmes dès la première année. Il explique, dans la préface du premier volume, son choix en soulignant le fait que les programmes des écoles italiennes les prévoient explicitement. Il spécifie, en outre, qu'il a choisi des phrases et des textes qui ont un rapport avec la vie réelle, qui ont un sens accompli et que ce ne sont jamais des phrases décousues. D'ailleurs, il ne faut pas oublier que 
Lovera a choisi de combiner plusieurs méthodes et il les applique dans son Corso. La présence d'exercices de traduction persiste dans les manuels pour un contrôle des connaissances lexicales et grammaticales des élèves et représente « un accroc à la méthode directe intégrale telle qu'elle est proclamée [...] » (Puren 2012 [1988] : 83).

\title{
TEMI.
}

\author{
1. Articolo e plurale dei nomi
}

A. Ecco la scuola. La scuola è grande; essa ha tre classi. Le classi sono grandi. Eceo la classe. Eceo il banco e la sedia. I banchi degli scolari sono puliti e comodi. I banchi sono per gli scolari e le scolare, la cattedra è per il maestro e per la maestra. La tavola nera è per il maestro e per la maestra, per gli scolari e per le scolare. Quali ogretti avete nella vostra classe? Nella nostra classe abbiamo la spugna, il gesso, le penne, i portapenne, i lapis, i quaderni. Abbiamo anche le righe, le squadre e i compassi. Chi ha il compasso? Gli scolari che imparano il disegno hanno un compasso o due compassi. Dove sono la riga e il temperino? La riga e il temperino sono nella cartella dello scolare. Gli scolari hanno le cartelle sotto ai banchi e i loro mantelli e cappelli sugli attaccapanni: essi hanno una carta sugante nei quaderni. Chi ha il giornale? Il professore ha due gior-
nali sulla cattedra.

Document 8 : Premier volume «Temi » $(1907: 116)$

Tous les termes présents dans ce thème ont été préalablement étudiés dans les exercices d'intuition et les élèves n'auront pas la nécessité de recourir au dictionnaire.

Les deux premiers volumes présentent seulement quelques pages de thèmes, le dernier volume possède un chapitre plus consistant et contient aussi des exercices de composition et des lettres commerciales.

\section{Conclusion}

Avec son manuel, Lovera a eu l'intention de faire connaître la langue française en tant qu' « organisme vital » en bannissant le lourd système « de la comparaison entre la langue étrangère et la langue maternelle » (Lovera 1907 : V).

L'enseignement intuitif représente un grand progrès dans l'enseignement linguistique ; c'est une méthode qui active la participation des élèves sans les ennuyer; son application est simple, elle abandonne la partie grammaticale lourde et ennuyeuse, et met la grammaire au service de la langue. Lovera synthétise de cette manière l'apport nouveau et dynamique de cette nouvelle méthode à l'enseignement des langues vivantes. Lovera a le mérite d'avoir lutté pour que la méthode directe et intuitive soit adoptée par les écoles italiennes, où la méthode grammaire-traduction représentait une institution intouchable pour la plupart des enseignants. Par le biais de son manuel et de ses articles dans le BFM, Lovera ébranla, sans toutefois le faire chavirer, le système cristallisé de l'enseignement des langues étrangères, en marquant un passage important dans l'histoire de l'école italienne. 


\section{Sources primaires}

(1904). Gymnasium : periodico letterario-didattico per le scuole secondarie. Anno 4. N.7.

(1910). L'écho français²2, 1-7. Palerme : Tip. Gazzetta Commerciale.

DENIKER, Josef (1904). Congrès international des langues vivantes, tenu à Paris, du 24 au 28 juillet 1900. Rapports, mémoires, liste des membres, etc., publiés par J. Deniker, Secrétaire général du congrès. Paris : Société pour la propagation des langues étrangères en France.

LOVERA, Romeo (dir.) (1894). Bollettino di Filologia Moderna : organo dell'Associazione nazionale tra i professori di lingue straniere. 15 janvier, Anno I, 1.

LOVERA, Romeo (dir.) (1901a). Bollettino di Filologia Moderna : organo dell'Associazione nazionale tra i professori di lingue straniere. Salò 28 febbraio, Anno III, 2.

LOVERA, Romeo (dir.) (1901b) Bollettino di Filologia Moderna : organo dell'Associazione nazionale tra i professori di lingue straniere. Venezia 31 ottobre, Anno III, 8.

LOVERA, Romeo (dir.) (1903a). Bollettino di Filologia Moderna : organo dell'Associazione nazionale tra i professori di lingue straniere. Venezia, Anno V, 1-2.

LOVERA, Romeo (dir.) (1903b). Bollettino di Filologia Moderna : organo dell'Associazione nazionale tra i professori di lingue straniere. Venezia Maggio-Giugno, Anno V, 9-10.

LOVERA, Romeo (1907). Corso di Lingua francese : Anno I. Palerme : , Libreria editrice del Bollettino di filologia Moderna.

LOVERA, Romeo (1910). Corso di Lingua francese a base intuitiva : Anno I. Rome-Milan : Società Ed. Dante Alighieri, Albrighi, Segati \& C.

LOVERA, Romeo (1919). Corso di Lingua francese a base intuitiva : Anno II. Rome : Albrighi, Segati \& $C$.

LOVERA, Romeo (1917). Corso di Lingua francese a base intuitiva : Anno III. Milan-Rome-Naples : Società Ed. Dante Alighieri, Albrighi, Segati \& C.

LOVERA, Romeo (1909). Fra Lingue e Letterature moderne (seconda serie). Rome-Milan : Società Editrice Dante Alighieri di Albrighi Segati \& C.

\section{Sources secondaires}

FINOTTI, Irene (2011). « Lexique et identité culturelle dans les manuels de la méthode directe ». Quaderni del CIRSIL, 9. Bologne : CLUEB. En ligne : [http://amsacta.unibo.it/2978/1/FINOTTI__2010.pdf].

GALAZZI, Enrica (2002). «Échos phonétiques en Sicile entre XIX ${ }^{\mathrm{e}}$ et $\mathrm{XX}^{\mathrm{e}}$ siècles ». Documents pour l'histoire du français langue étrangère ou seconde, 28, 13-31.

GERMAIN, Claude (1993). Évolution de l'enseignement des langues : 5000 ans d'histoire. Paris : Nathan/ CLE. 
LENTINI, Rosario (2012). « L'istituzione della scuola media di commercio a Palermo tra '800 e '900 », Mediterranea. Ricerche storiche, 26, 475-502.

MINERVA, Nadia (1996). Manuels, maîtres, méthodes. Repères pour l'histoire de l'enseignement du français en Italie. Bologne : CLUEB.

MINERVA, Nadia (2005). « Le rôle des revues spécialisées dans la formation des enseignants de français de l'Italie post-unitaire (1883-1915) ». Documents pour l'histoire du français langue étrangère ou seconde, 33/34, 197-213.

PELLANDRA, Carla (2004). Le radici del nostro mestiere. Storia e storie degli insegnamenti linguistici.

Quaderni del CIRSIL 3, 1-152. Bologne : CLUEB.

PUREN, Christian (2012 [1988]). Histoire des méthodologies de l'enseignement des langues. Paris : CLE international. Version numérisée en ligne : [https://www.christianpuren.com/mes-travaux/ $1988 \mathrm{a} /]$.

RAVA CORINALDI, Bice (1907). L'insegnamento delle lingue viventi nelle scuole medie. Rome-Milan :

Società Ed. Dante Alighieri, Albrighi, Segati \& C.

ROSSIGNOTTI, Giovanna (1991). « La didattica della lingua francese attraverso l'analisi del Bollettino di Filologia Moderna (1894-95 ; 1901-1909) ». Documents pour l'histoire du français langue étrangère ou seconde, 8, 265-276.

\section{NOTES}

1. La traduction des textes italiens est de notre fait.

«[...] ci viene dalla Germania dove, nel 1882 appare il libello di un professore di filologia anglosassone dell'Università di Marburgo, Wilhelm Viëtor, che porta un titolo eloquente : Der Sprachunterricht muss umkehren (l'insegnamento delle lingue deve cambiare radicalmente) ».

2. « utile e qualificante per portare a compimento il suo progetto didattico ».

3. Le Bollettino a également attiré l'attention d'Enrica Galazzi qui a analysé l'impact du mouvement de la réforme sur Lovera et ses collaborateurs, les ouvertures du professeur de Salò aux études phonétiques, sa chronique, dans les pages du Bollettino, des articles du Maître phonétique et ses comptes rendus des ouvrages de Passy.

4. «[...] intende presentare al pubblico [...] una lista delle pubblicazioni filologiche [...], aggiungendovi i propri apprezzamenti ispirati sempre al più sincero convincimento ed a quella pratica utilità che dalle lingue estere si dovrebbe ricavare ».

5. « la lingua viva e non fossilizzata ».

6. « Il metodo diretto richiede costante attenzione, da parte dei discenti, e profonda conoscenza della lingua estera da parte dei docenti : due buone qualità che ancora fanno in parte difetto in Italia ».

7. «Prima si impari a parlare, poi a leggere, poi a scrivere » (Manfredini, anno IV, dicembre 1904, n. 7, Gymnasium, 123).

8. « questi metodi hanno tutti una base pressoché comune nei principi che guidano l'associazione fonetica, il cui presidente Viëtor [...] è il più ardente propugnatore della riforma dell'insegnamento linguistico » (1901). BFM, année III, 8 : 7.

9. Nous avons analysé les éditions de 1907, 1910, 1917 et 1919.

10. Une ample liste, par ordre alphabétique, des villes italiennes est publiée à la fin du numéro 3-4 de L'écho français du 15, 28 février 1910. Parmi les différentes villes certaines sont mises en évidence en caractères gras: Bari, Catania, Cremona, Cuneo, Genova, Grosseto, Livorno, Macerata, Mantova, Milano, Palermo, Pavia, Perugia, Pesaro, Pisa, Potenza, Ravenna, Reggio Calabria, Roma, Siracusa, 
Sondrio, Torino, Venezia et Verona. Cela nous permet d'avoir une vision complète du succès de son manuel et de ses ouvrages en général.

11. La République italienne est fondée sur les résultats du referendum institutionnel du 2 juin 1946.

12. «La prima cura del maestro deve essere di familiarizzare gli allievi coi suoni della lingua straniera. A questo scopo egli si varrà di una trascrizione fonetica, che sarà impiegata in luogo dell'ortografia tradizionale durante la prima parte del corso ».

13. Voir aussi Lovera (1903a).

14. Romeo Lovera spécifie, dans la préface, qu'il a choisi les tableaux muraux édités par la Librairie Armand Colin de Paris.

15. «I quadri murali per uso dell'insegnamento delle lingue sono ancora poco diffusi fra noi ; essi cominciano per altro ad essere introdotti nelle scuole, e l'Italia conta anch'essa i suoi autori di quadri nei professori Lovera e Manfredini, che ne stanno pubblicando una serie a tale scopo ».

16. «Ils doivent leur nom à la maison d'édition viennoise chargée de leur production » (Rossignotti, $1990: 271$, note 22).

17. «Col ricavare induttivamente la grammatica dai testi studiati, la si renderà realmente utile $\mathrm{e}$ gradita, poiché lo studioso vedrà che essa è infatti la codificazione di leggi del linguaggio, e non una raccolta di dogmi oscuri, educatori d'un popolo di pappagalli ».

18. « in grandi ma complesse linee, sì da formarne [...] una catena di regole chiare e sistematicamente ordinate ».

19. Professeur au Real Ginnasio de Sezze et auteur de nombreux articles publiés dans le BFM.

20. « un ragazzo nostro dagli 8 ai 10 anni comprende il suo libro di lettura senza afferrarne tutto il senso nei suoi penetrali » (Lovera $1903 \mathrm{~b}: 142)$.

21. « si è obbligati a 'jeter sa langue aux chiens' « (Lovera $1903 \mathrm{~b}: 143$ ).

22. La Revue paraît deux fois par mois, excepté en août et en septembre.

\section{RÉSUMÉS}

Nous analysons les trois volumes du manuel de Romeo Lovera, Corso di Lingua francese a base intuitiva, destiné à l'enseignement de la langue française dans les écoles secondaires italiennes. Romeo Lovera est le plus grand partisan, en Italie, de la réforme méthodologique de l'enseignement des langues étrangères. Dans ses manuels, dès la première leçon, à travers l'intuition directe et indirecte de la langue étrangère, l'élève apprend à parler la langue française en étudiant les expressions et les phrases les plus usuelles et en apprenant la grammaire de manière inductive.

We analyze the three volumes of Romeo Lovera's manual, Corso di Lingua francese a base intuitiva, which were intended for teaching French in Italian secondary schools. Romeo Lovera is Italy's biggest supporter of methodological reform in the teaching of foreign languages. In their manuals, from the first lesson, through the direct and indirect intuition of the foreign language, the student learns to speak the French language by studying the most usual expressions and sentences and by learning grammar inductively. 
INDEX

Keywords : French language teaching, Corso di Lingua francese a base intuitiva, direct method, intuitive method, Romeo Lovera

Mots-clés : enseignement de la langue française, Corso di Lingua francese a base intuitiva, méthode directe, méthode intuitive, Romeo Lovera

\section{AUTEUR}

MARIE-DENISE SCLAFANI

Université de Palerme mariedenise.sclafani@unipa.it 